\title{
AN EXAMPLE OF A HILBERT RING WITH MAXIMAL IDEALS OF DIFFERENT HEIGHT
}

\author{
LESLIE G. ROBERTS
}

\begin{abstract}
An example is given of an integral domain which is a Hilbert ring of Krull dimension two but has some maximal ideals of height one.
\end{abstract}

1. Introduction. Examples of integral domains which are Hilbert rings with maximal ideals of different height do-not seem to be well known. In [3, §5, Example 2], Krull has an example of a Hilbert ring of dimension 2 with one maximal ideal of height 1 that is obtained by localization from $K\left[X_{1}, X_{2}\right]$, where $K$ is a countable field. In this paper, by localization from $C\left[X_{1}, X_{2}\right]$, I obtain a Hilbert ring of dimension 2 with an uncountable number of maximal ideals of height 1 . By further localization one can obtain a Hilbert ring of dimension 2 with any prescribed finite (or countable) number of maximal ideals of height 1 .

By Hilbert ring I mean a ring in which every prime ideal is the intersection of maximal ideals. The height of a prime ideal $P$ is the length $n$ of the longest chain $0 \subseteq P_{0} \subsetneq P_{1} \subsetneq P_{2} \subsetneq \cdots \subsetneq P_{n}=P$ of prime ideals from 0 to $P$. Note that if the ring is not required to be an integral domain, then one can take a reducible variety with components of different dimensions. $R$ and $C$ denote the real and complex numbers, respectively.

I learned of the problem from [1, p. 4].

2. The example. Let $A=C[Z, W]$, where $Z$ and $W$ are indeterminants. Identify $C^{2}=\{(z, w) \mid z, w \in C\}$ with $R^{4}=\left\{\left(x_{0}, x_{1}, x_{2}, x_{3}\right) \mid x_{i} \in R\right\}$ by $z=$ $x_{0}+i x_{1}, w=x_{2}+i x_{3}$. Let $U=\left\{\left.(z, w)|| z\right|^{2}+|w|^{2}<1\right\}$. This is just the interior of the unit ball in $R^{4}$. Let $S$ be the multiplicative set in $A$ generated by all linear polynomials $a Z+b W+c$ whose variety does not meet $U$. Then, I claim that $A_{S}$ is a Hilbert ring of dimension 2 that has an infinite number of maximal ideals of height 1 .

Spec $A_{S}$ is the subset of Spec $A$ consisting of all prime ideals that do not meet $S$. First of all, every closed point $P$ in Spec $A$ lying outside of $U$ lies on the variety of some element of $S$. For, let $P=\left(z_{0}, w_{0}\right)$ with $\left|z_{0}\right|^{2}+$ $\left|w_{0}\right|^{2} \geqq 1$. Then the complex line $\bar{z}_{0} Z+\bar{w}_{0} W=\left|z_{0}\right|^{2}+\left|w_{0}\right|^{2}$ contains $P$ and

Received by the editors April 12, 1972.

AMS (MOS) subject classifications (1970). Primary 13A15.

(c) American Mathematical Society 1973 
does not meet $U$, for its real part is just the hyperplane in $R^{4}$ through $P$ perpendicular to the line from the origin to $P$. Thus all closed points in Spec $A$ outside $U$ have been eliminated so the maximal ideals in $A_{S}$ of height 2 correspond to the points of $U$. On the other hand, there exist curves in $C^{2}$ which are not a product of complex lines and do not meet $U$, for any curve not passing through the origin is a positive distance away from the origin, and multiplying all coordinates by a sufficiently large real constant gives a curve missing $U$. These correspond to maximal ideals of height 1 in $A_{S}$. Finally, $A_{S}$ is a Hilbert ring. To show this, one must prove that every prime of height 1 that is not maximal is contained in infinitely many maximal ideals (by Theorem 147 of [2]). But such ideals correspond to curves in $C^{2}$ that meet $U$. Any such curve meets $U$ in an infinite number of points because $U$ is open, and hence the corresponding ideal is contained in an infinite number of maximal ideals.

In $A_{S}$ all the maximal ideals of height 1 are principal. Let $S^{\prime}$ be a set of generators of all but a finite (or countable) number of these ideals. We may choose $S^{\prime}$ so that $S^{\prime} \subset A$. Let $T=S \cup S^{\prime}$. Then $A_{T}$ will be a Hilbert ring of dimension 2 with a prescribed finite (or countable) number of maximal ideals of height 1 .

Finally $A_{S}\left[X_{1}, \cdots, X_{n}\right]$ (respectively $A_{T}\left[X_{1}, \cdots, X_{n}\right]$ ) is a Hilbert ring of dimension $n+2$ with an uncountable (respectively countable or finite) number of maximal ideals of height $n+1$. I do not know if a Krull ring of dimension $n$ can have maximal ideals of height less than $n-1$.

\section{REFERENCES}

1. A. V. Geramita, Maximal ideals in polynomial rings over Dedekind domains, Queen's University Mathematical Preprint No. 1971-20.

2. I. Kaplansky, Commutative rings, Allyn \& Bacon, Boston, Mass., 1970. MR 40 \#7234.

3. W. Krull, Jacobsonsche Ringe, Hilbertscher Nullstellensatz, Dimensiontheorie, Math. Z. 54 (1951), 354-387. MR 13, 903.

Department of Mathematics, Queen's University, Kingston, Ontario, Canada 